\title{
Use of 5-Aminolevulinic Acid to Detect Residual Meningioma and Ensure Total Removal while Avoiding Neurological Deficits
}

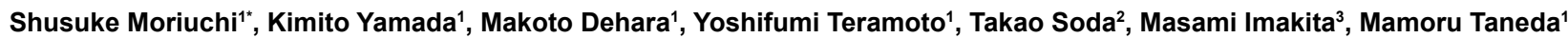

${ }^{1}$ Departments of Neurosurgery, Rinnku General Medical Center, Izumisano, Osaka, Japan

${ }^{2}$ Departments of Neurology, Rinnku General Medical Center, Izumisano, Osaka, Japan

${ }^{3}$ Departments of 3Pathology, Rinnku General Medical Center, Izumisano, Osaka, Japan

\begin{abstract}
5-Aminolevulinic acid (5-ALA) has been used successfully to resect meningioma without leaving a residual mass. The authors report their experience resecting meningiomas in 17 patients using 5-ALA. Except for one case, all meningiomas fluoresced intra-operatively under the microscope. Invasion to the dura mater, brain parenchyma, or skull showed fluorescence, allowing for confirmation of residual tumor; total removal of the meningioma could be performed more easily, and unexpected neurological deficits could be prevented by precise removal of the tumor under the microscope. With invasion to the dura mater or skull in one case, the extent of dural removal was decided by 5-ALA fluorescence with 1- to 2-cm safety margins. In another case with parenchymal invasion, close removal of the tumor without leaving residual tumor could be performed with 5-ALA fluorescence. With the above methods, no serious side effects or complications occurred in this study. Not all meningiomas fluoresced with 5-ALA, and 5-ALA is available for about $95 \%$ of meningiomas. 5-ALA appears easy to use and helpful for finding residual tumor and preventing recurrences by total removal of meningiomas.
\end{abstract}

Keywords: 5-ALA; Meningioma; Total resection

\section{Introduction}

Complete resection of meningiomas provides patients with the best chance for a cure; however, surgery is frequently difficult given the proximity of lesions to vital structures, such as cranial nerves, major vessels, and venous sinuses [1]. Accurate discrimination between tumor and normal tissue is crucial for optimal tumor resection. With the use of 5-aminolevulinic acid (ALA), meningiomas can be seen to fluoresce intra-operatively under the microscope [2]. Invasion to the dura mater, brain parenchyma, or skull shows fluorescence, allowing for confirmation of residual tumor, and total removal of the meningioma could be performed more easily, while unexpected neurological deficits could be prevented by precise removal of the tumor under the microscope. In the past 2 years, 17 total resections of meningiomas were performed using 5-ALA without recurrences and major complications, and it was found that fluorescence-guided resection may be beneficial for removal of complicated meningiomas that have a high risk of recurrence.

\section{Methods}

\section{Patients' characteristics}

A total of 17 consecutive patients (16 females, 1 male; average age, 65.5 years) undergoing resection of intracranial meningiomas from January 2011 to December 2012 were included in this study (Table 1). All of the meningiomas were histologically Grade I meningiomas. Tumor locations varied and included parasagittal, falx, sphenoid ridge, convexity, planum sphenoidale, and petroclival tumors. The sizes of the meningiomas ranged from more than $21 \mathrm{~mm}$ in diameter (Case 7) to a maximum of $76 \mathrm{~mm}$ in diameter (Case 2). In 4 of 17 cases, the middle meningeal artery feeding the meningioma was embolized preoperatively.

\section{Preoperative and intraoperative procedures}

After confirmation of normal liver function, patients were given 20 $\mathrm{mg} / \mathrm{kg}$ of 5-ALA (Cosmo Oil Co., Ltd., Japan) 4 hours preoperatively $[2,3]$. Craniotomies were performed under general anesthesia. The meningiomas were confirmed using a 440-nm ultraviolet light source (violet-blue light), an optical component of the OPMI Pentero microscope (Carl Zeiss AG, Germany). Under the violet-blue light, the meningiomas showed charcoal-red fluorescence. Total removal of the meningiomas could be performed by resecting the tumor until the charcoal-red fluorescence could no longer be detected. In cases of proximity to vital organs, it is important to prevent damage to these organs and carefully remove the charcoal-red residual tumors.

\section{Results}

\section{Tumor fluorescence}

All 17 tumors were totally resected using 5-ALA fluorescence. No major neurological deficits were observed after surgery. The histopathological diagnosis of these 17 meningiomas was WHO grade 1 meningioma; the tumors' MIB-1 indices did not reach 5\%. At the latest follow-up examination, 2 years after surgery, no patients showed evidence of recurrence. In 4 cases, the middle meningeal artery was embolized preoperatively. Even when the main feeding artery, the middle meningeal artery, was embolized, tumor fluorescence was strong in all cases. No correlation between preoperative embolization and tumor fluorescence was observed. Two cases (Case 4 and Case 5) showed weak tumor fluorescence. In Case 4, the tumor had intratumoral hemorrhage. The histopathological diagnoses were meningothelial meningioma with a MIB- 1 index of $2 \%$ in Case 4 and transitional meningioma with a

*Corresponding author: Dr. Shusuke Moriuchi, Departments of Neurosurgery, Rinnku General Medical Center, Izumisano, Osaka, Japan, Tel: 81-72-469-3111; Fax: 81-72-469-7929; E-mail: s-moriuchi@rgmc.izumisano.osaka.jp

Received June 21, 2013; Accepted July 29, 2013; Published August 05, 2013

Citation: Moriuchi S, Yamada K, Dehara M, Teramoto Y, Soda T, et al. (2013) Use of 5-Aminolevulinic Acid to Detect Residual Meningioma and Ensure Total Removal while Avoiding Neurological Deficits. J Neurol Neurophysiol 4: 159. doi:10.4172/2155-9562.1000159

Copyright: (c) 2013 Moriuchi S, et al. This is an open-access article distributed under the terms of the Creative Commons Attribution License, which permits unrestricted use, distribution, and reproduction in any medium, provided the original author and source are credited. 
MIB-1 index of $1 \%$ in Case 5 . No correlation between size and tumor fluorescence was observed.

\section{Representative cases}

Case 3: A 37-year-old woman presented with headache and mild right hemiparesis. MR imaging showed a large meningioma (maximum diameter $59 \mathrm{~mm}$ ) at the left parasagittal area, extending to the skull and causing the skull to bulge (Figure 1A). After craniotomy, it was found that the meningioma invaded into the inner layer of the skull and fluoresced charcoal-red (Figure 1B). The invasion of meningioma into the inner layer was drilled out until the charcoal-red light disappeared, and the skull was returned at closure. The parasagittal meningioma fluoresced strongly and was removed totally, and the attachment to the lateral wall of the superior sagittal sinus was coagulated (Figures 1C and 1D). The patient had no neurological deficit at discharge. The histopathological diagnosis was transitional meningioma with a MIB-1 index of $2 \%$.

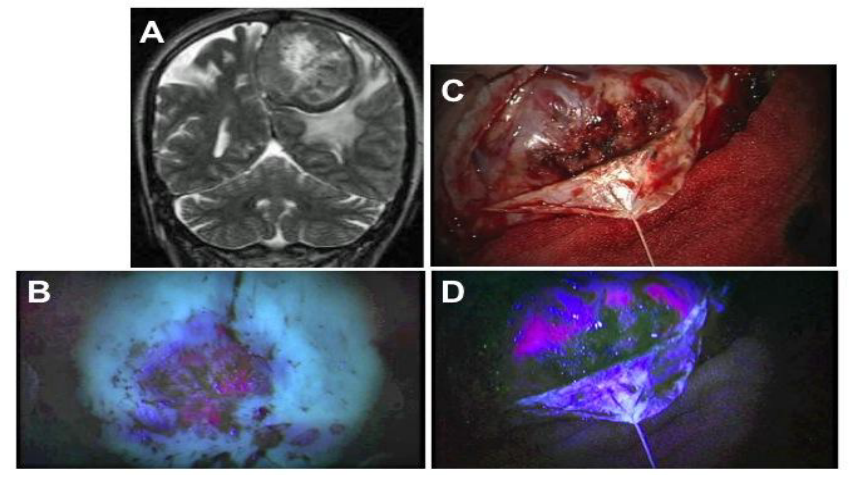

Figure 1: A. Case 1. Preoperative, axial, T1-weighted, Gd-enhanced, MR image demonstrating a well-marginated, enhanced tumor in the left thalamus. B. Case 1. Postoperative plain CT demonstrating a small hemorrhage in the left thalamus after stereotactic biopsy (arrow)

C. Case 2. Preoperative, axial, T2-weighted, MR image demonstrating a massive, diffuse, pontine glioma.

D. Postoperative, axial, T2-weighted, MR image demonstrating a high-intensity area after stereotactic biopsy (arrow).

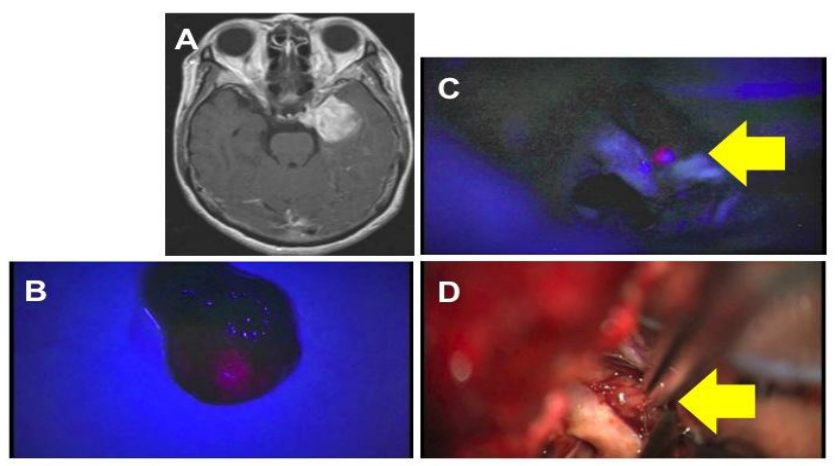

Figure 2: $A, B$. Case 1. Tumor specimen of malignant lymphoma under white light illumination $(A)$ and under a violet-blue light (B). One pair of tumor specimens at the left shows charcoal-red fluorescence; in contrast, the negative control of subcutaneous tissue at right shows no fluorescence (B).

C,D. Case 2. Tumor specimen of pontine glioma under white illumination (C) and under a violet-blue light (D). One pair of tumor specimens at left shows slight charcoal-red fluorescence; in contrast, the negative control of subcutaneous tissue at right shows no fluorescence (D).

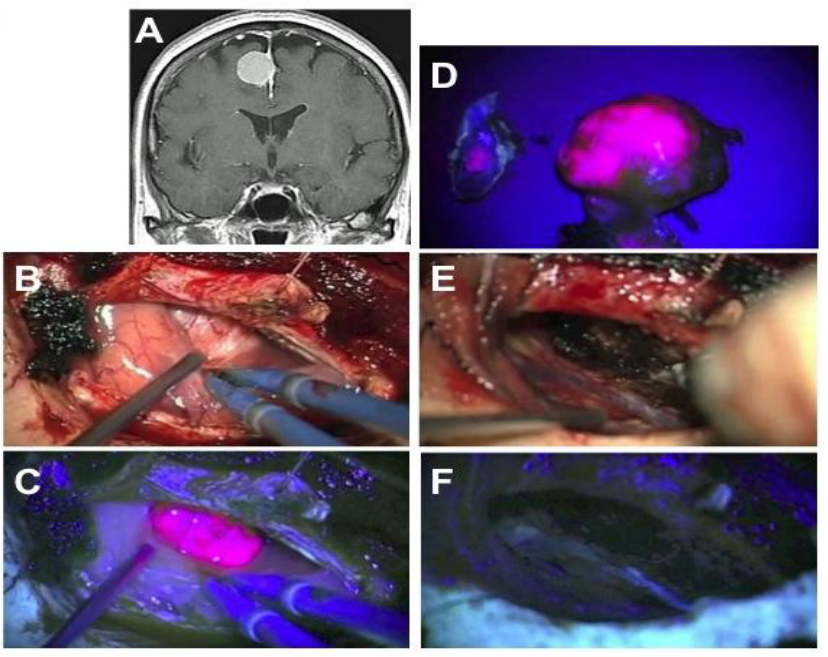

Figure 3: Photomicrograph of samples from patients.

$A, B$. Case 1. A: The lesion is a diffuse large B-cell lymphoma. $H$ and $E$, original magnification $\times 200$. B, MIB-1 index $24 \%$. MIB-1 staining $\times 400$.

$\mathrm{C}, \mathrm{D}$. Case 2. C: The lesion is a diffuse grade 2 astrocytoma with a MIB-1 index of $12 \% . \mathrm{H}$ and $\mathrm{E}, \mathrm{x} 200$ (C). MIB-1 staining, $\mathrm{x} 200$ (D)

Case 4: A 67-year-old woman presented with diplopia and headache. MR imaging showed a left sphenoid ridge meningioma with intratumoral hemorrhage and parenchymal edema adjacent to the tumor (maximum diameter $44 \mathrm{~mm}$ ) (Figure 2A). The tumor encased the left middle cerebral artery and invaded into the brain parenchyma with charcoal-red fluorescence (Figures $2 \mathrm{~B}$ and 2D). The tumor was resected totally until no fluorescence was observed. The patient had no neurological deficit and no epileptic seizures after operation. The histopathological diagnosis was meningothelial meningioma with a MIB-1 index of $2 \%$.

Case 10: A 59-year-old woman presented with headache and mild weakness of the right lower extremity. MR imaging showed a right falx meningioma (maximum diameter $30 \mathrm{~mm}$ ) (Figure $3 \mathrm{~A}$ ). The tumor showed strong charcoal-red fluorescence (Figure 3B-D). The falx attached to the tumor was coagulated, and the fluorescence disappeared (Figures $3 \mathrm{E}$ and $3 \mathrm{~F}$ ). After the attached falx was resected, the resected tumor and the opposite side of the falx to which the tumor was attached showed strong charcoal-red fluorescence (Figure 3D), showing that the meningioma invaded through the falx. The headache and weakness of the lower extremity disappeared after total resection. The histopathological diagnosis was transitional meningioma with a MIB-1 index of $2 \%$.

\section{Discussion}

In this study, 5-ALA administration resulted in bright and diffuse tumor fluorescence in 15 (88\%) of 17 cases, including the cases in which pre-operative embolization had been performed (Table 1). Protoporphyrin IX (PPIX) fluorescence was seen only in the main mass and areas of tumor invasion. In this series, the sensitivity and specificity of PPIX fluorescence of the main tumor mass were $88 \%$ (15 of 17 cases) and $100 \%$ (17 of 17 cases), respectively (Table 1). Fluorescence guidance allowed us to identify the extent of the tumor and helped us avoid leaving residual tumor tissue that was difficult to identify in the white-light mode. If we had not used fluorescence guidance, we might not have noticed several small areas of residual tumor showing 5-ALA fluorescence under violet-blue light with an operative microscope in 
the operating room. In this study, numerous factors affected tumor recurrence: the tumor's soft consistency, lobular shape, encasement of the artery, invasion into the brain parenchyma, and dural attachment very close to the venous sinus, as well as bone invasion. Because of these factors, the risk of recurrence was considered very high [4-7]. It is worth noting that tumor remnants were identified by fluorescence in multiple regions. deVries and Wakhloo [8] reported that recurrent tumor is often found at multiple sites. Meningioma has a high risk of recurrence, and during excision, meningioma tissue can be left at any attachment to surrounding tissues, especially at attachments to the gliotic brain, major sinuses, the anterior visual pathway [9], and marginal dura mater. Aggressive excision of the dura and gliotic brain has been recommended to reduce this risk $[7,10]$, but the optimal extent of dural resection has been controversial. Kinjyo et al. proposed a margin of 2 $\mathrm{cm}$. Nakasu et al. reported that a $1-\mathrm{cm}$ dural margin is insufficient to prevent recurrence $[11,7]$. The most suitable margin for dural excision will necessarily differ from case to case, because of differences in tumor growth rates and invasiveness. In light of these factors, photodynamic diagnosis may become a promising method of determining the extent of dural resection.

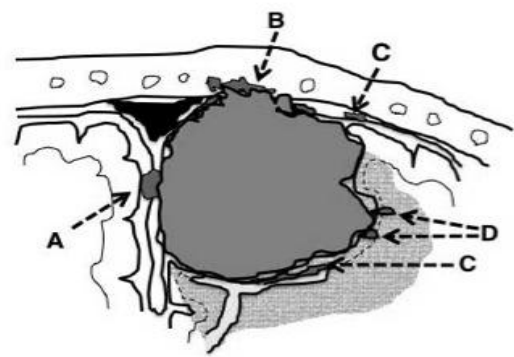

Figure 2. A,B. Case 1. Tumor specimen of malignant lymphoma under white light illumination $(A)$ and under a violet-blue light $(B)$. One pair of tumor specimens at the left shows charcoal-red fluorescence; in contrast, the negative control of subcutaneous tissue at right shows no fluorescence (B).

C,D. Case 2. Tumor specimen of pontine glioma under white illumination (C) and under a violet-blue light (D). One pair of tumor specimens at left shows slight charcoal-red fluorescence; in contrast, the negative control of subcutaneous tissue at right shows no fluorescence (D).
On the other hand, indiscriminate excision can lead to complications related to brain and vascular injury. Therefore, if a tumor is located close to a major sinus, the skull base, or an eloquent area, then the excision of dura mater and brain tissue should be restricted to safe areas, and unresectable dura must be coagulated. Fluorescence guidance may help avoid unnecessary excision. The reasons why tumor remnants may be overlooked can be classified into three categories. 1) The tumor cells invade surrounding tissue, attached dura mater, bone, and brain, after which the invaded area is difficult to distinguish from the noninvaded area (Figure 4). 2) The tumor remnants may be hidden behind large vessels or the sinus, dural fold, or sulcus (Figure 4). 3) Daughter lesions may develop apart from the main mass. All three reasons can make tumor remnants difficult to identify with the naked eye or with the aid of a surgical microscope. The use of 5-ALA-induced fluorescence can help surgeons identify tumor remnants, because an area of strong red fluorescence will appear if even a small part of a remnant is present at the tissue surface. Fluorescence can make it easy to distinguish the one from the other and determine the extent of a given tumor.

In the present study, tumor invasion into the skull was also visualized by charcoal-red fluorescence. In most cases, hyperostosis in association with meningioma is related to tumor invasion. However, the extent of tumor invasion is difficult to judge from the appearance alone. Although it is relatively easy to treat bone invasion in cases of convexity meningioma, invasion at the skull base is hard to treat because it involves the cranial nerves, major blood vessels, and air sinuses. Therefore, the detection of bone invasion using photodynamic diagnostic methods would seem to be valuable, especially in surgery for skull-base meningiomas.

Many recent reports have described the usefulness of 5-ALA to identify the margin of a malignant tumor or glioma for maximal cytoreduction [6,12-16]. 5-ALA is an endogenous body metabolite central to heme biosynthesis that is readily absorbed and metabolized into porphyrins by malignant tumor cells (5). This phenomenon can aid in tumor resection to identify the residual tumor in tumor margins, when gross total resection is possible and desirable, with malignant cells fluorescing, allowing discrimination between tumor and normal functional brain tissue $[6,12,13]$. The side effects of 5-ALA are mild. Skin irritation, nausea, and transient elevation in liver function test

\begin{tabular}{|c|c|c|c|c|c|}
\hline Case No. & Age, Sex & Tumor Location & Tumor Diameter(mm) & Tumor Fluorescence & Preoperative Embolization \\
\hline 1 & $62, \mathrm{~F}$ & rt PO parasagittal & $44 \times 40 \times 46$ & Strong & No \\
\hline 2 & $62, F$ & rt F falx & $76 \times 49 \times 67$ & Strong & Yes \\
\hline 3 & $37, \mathrm{~F}$ & It FP parasagittal & $59 \times 58 \times 50$ & Strong & Yes \\
\hline 4 & $67, F$ & It sphenoid ridge & $44 \times 38 \times 40$ & Weak & No \\
\hline 5 & $45, \mathrm{~F}$ & It petroclival & $28 \times 14 \times 24$ & Weak & No \\
\hline 6 & $65, M$ & rt $\mathrm{P}$ convexity & $25 \times 19 \times 26$ & Strong & No \\
\hline 7 & $75, \mathrm{~F}$ & rt sphenoid ridge & $21 \times 21 \times 20$ & Strong & No \\
\hline 8 & $65, F$ & It sphenoid ridge & $63 \times 46 \times 59$ & Strong & Yes \\
\hline 9 & $74, \mathrm{~F}$ & It $P$ convexity & $40 \times 40 \times 46$ & Strong & No \\
\hline 10 & $59, \mathrm{~F}$ & rt $P$ falx & $30 \times 25 \times 21$ & Strong & Yes \\
\hline 11 & $75, F$ & planum sphenoidale & $37 \times 33 \times 31$ & Strong & No \\
\hline 12 & $81, F$ & It $\mathrm{F}$ convexity & $41 \times 29 \times 28$ & Strong & No \\
\hline 13 & $60, F$ & rt petroclival & $26 \times 24 \times 27$ & Strong & No \\
\hline 14 & $48, F$ & planum sphenoidale & $22 \times 19 \times 20$ & Strong & No \\
\hline 15 & $60, F$ & rt F convexity & $26 \times 18 \times 23$ & Strong & No \\
\hline 16 & $64, \mathrm{~F}$ & rt sphenoid ridge & $32 \times 26 \times 26$ & Strong & No \\
\hline 17 & $48, F$ & It FP parasagittal & $42 \times 32 \times 26$ & Strong & No \\
\hline
\end{tabular}

Table 1. Characteristics of patients with meningioma. Abbreviations used in this table: M, male; F, female; PO, parieto-occipital; F, frontal; $P$, fronto-parietal; $P$, parietal; rt, right; It, left. 
results have occurred in some adult patients given higher doses of 5-ALA $[4,8,17,18]$. Apart from transient nausea after dye ingestion, the present patients had no side effects, and their liver function tests were unchanged.

Accumulation in the normal central nervous system is restricted except in the subependymal zone and choroid plexus [18,19]. Many possible mechanisms have been posited to explain the selective accumulation of PPIX in neoplasms: (1) enhanced penetration of 5-ALA through the blood-brain barrier; (2) reduced transporter activity that drains PPIX outside of cells; and (3) reduced activity of ferrochelatase, which converts PPIX to heme [20]. At least one of these factors is probably involved in the strong 5-ALA-derived fluorescence in meningiomas. In general, there is no strict correlation between cell proliferation and PPIX accumulation. In meningiomas, the proliferation rate is relatively low [12].

In conclusion, applying this method to meningiomas that have a high risk of recurrence should be of value not only in ensuring that tumor remnants are not overlooked during resection but also in helping to avoid unnecessarily radical resection and the associated risk of morbidity. To confirm the usefulness of fluorescence-guided surgery for meningioma, further studies on its sensitivity, specificity, and effect on recurrence rates are needed.

\section{Disclaimer}

The authors report no conflict of interest concerning the materials or methods used in this study or the findings reported in this paper.

\section{References}

1. Samii M, Gerganov VM (2008) Surgery of extra-axial tumors of the cerebral base. Neurosurgery 62: 1153-1166.

2. Kajimoto Y, Kuroiwa T, Miyatake S, Ichioka T, Miyashita M, et al. (2007) Use of 5 -aminolevulinic acid in fluorescence-guided resection of meningioma with high risk of recurrence. Case report. J Neurosurg 106: 1070-1074.

3. Moriuchi S, Yamada K, Dehara M, Teramoto Y, Soda T, et al. (2011) Use of 5-aminolevulinic acid for the confirmation of deep-seated brain tumors during stereotactic biopsy. Report of 2 cases. J Neurosurg 115: 278-280.

4. Al-Mefty O, Kadri PA, Pravdenkova S, Sawyer JR, Stangeby C, et al. (2004) Malignant progression in meningioma: documentation of a series and analysis of cytogenetic findings. J Neurosurg 101: 210-218.

5. Jääskeläinen J (1986) Seemingly complete removal of histologically benign intracranial meningioma: late recurrence rate and factors predicting recurrence in 657 patients. A multivariate analysis. Surg Neurol 26: 461-469.

6. Jääskeläinen J, Haltia M, Servo A (1986) Atypical and anaplastic meningiomas: radiology, surgery, radiotherapy, and outcome. Surg Neurol 25: 233-242.

7. Nakasu S, Nakasu Y, Nakajima M, Matsuda M, Handa J (1999) Preoperative identification of meningiomas that are highly likely to recur. J Neurosurg 90 455-462.

8. de Vries J, Wakhloo AK (1994) Repeated multifocal recurrence of grade I, grade II, and grade III meningiomas: regional multicentricity (primary new growth) or metastases? Surg Neurol 41: 299-305.

9. Stafford SL, Perry A, Suman VJ, Meyer FB, Scheithauer BW, et al. (1998) Primarily resected meningiomas: outcome and prognostic factors in 581 Mayo Clinic patients, 1978 through 1988. Mayo Clin Proc 73: 936-942.

10. Salcman M (1991) Malignant meningiomas, in Al-Mefty O (ed): Meningiomas New York: Raven Press, 75-86

11. Kinjo T, al-Mefty O, Kanaan I (1993) Grade zero removal of supratentoria convexity meningiomas. Neurosurgery 33: 394-399.

12. linuma S, Farshi SS, Ortel B, Hasan T (1994) A mechanistic study of cellular photodestruction with 5-aminolaevulinic acid-induced porphyrin. $\mathrm{Br} \mathrm{J}$ Cancer 70: 21-28.

13. Kallio M, Sankila R, Hakulinen T, Jääskeläinen J (1992) Factors affecting operative and excess long-term mortality in 935 patients with intracranial meningioma. Neurosurgery 31: 2-12.

14. Morofuji Y, Matsuo T, Hayashi Y, Suyama K, Nagata I (2008) Usefulness of intraoperative photodynamic diagnosis using 5-aminolevulinic acid for meningiomas with cranial invasion: technical case report. Neurosurgery 62 102-103.

15. Coluccia D, Fandino J, Fujioka M, Cordovi S, Muroi C, et al. (2010) Intraoperative 5 -aminolevulinic-acid-induced fluorescence in meningiomas. Acta Neurochir (Wien) 152: 1711-1719.

16. Bekelis K, Valdés PA, Erkmen K, Leblond F, Kim A, et al. (2011) Quantitative and qualitative 5-aminolevulinic acid-induced protoporphyrin IX fluorescence in skull base meningiomas. Neurosurg Focus 30: E8.

17. Borovich B, Doron $Y(1986)$ Recurrence of intracranial meningiomas: the role played by regional multicentricity. J Neurosurg 64: 58-63.

18. Ennis SR, Novotny A, Xiang J, Shakui P, Masada T, et al. (2003) Transport of 5-aminolevulinic acid between blood and brain. Brain Res 959: 226-234.

19. Olivo M, Wilson BC (2004) Mapping ALA-induced PPIX fluorescence in norma brain and brain tumour using confocal fluorescence microscopy. Int $\mathrm{J}$ Oncol 25: $37-45$.

20. Peng Q, Warloe T, Berg K, Moan J, Kongshaug M, et al. (1997) 5-Aminolevulinic acid-based photodynamic therapy. Clinical research and future challenges. Cancer 79: 2282-2308. 